\begin{tabular}{c} 
Journal of Mathematical Sciences and Modelling, 2(1) (2019) 1-13 \\
Research Article \\
Journal of Mathematical Sciences and Modelling \\
Journal Homepage: www.dergipark.gov.tr/jmsm \\
ISSN 2636-8692 \\
DOI: http://dx.doi.org/10.33187/jmsm.416628 \\
\hline
\end{tabular}

\title{
Another Two-Parameter Sujatha Distribution with Properties and Applications
}

\author{
Mussie Tesfay ${ }^{1}$ and Rama Shanker ${ }^{1 *}$ \\ ${ }^{1}$ Eritrea Institute of Technology, Asmara, Eritrea \\ * Corresponding author
}

\section{Article Info}

Keywords: Applications, Estimation of parameters, Exponential distribution, Moments, Statistical properties, Sujatha distribution.

2010 AMS: 62E05, 62E99.

Received: 18 April 2018

Accepted: 29 November 2018

Available online: 20 April 2019

\begin{abstract}
In this paper another two-parameter Sujatha distribution (ATPSD), which includes exponential distribution and Sujatha distribution as particular cases, has been proposed. Statistical properties including shapes for varying values of parameters, moments, coefficient of variation, skewness, kurtosis, index of dispersion, hazard rate function, mean residual life function, stochastic ordering, mean deviations, Bonferroni and Lorenz curves, and stressstrength reliability of ATPSD have been discussed. The method of moment estimation and the method of maximum likelihood estimation have been discussed for estimating its parameters. Finally, applications of ATPSD have been discussed with two real lifetime datasets.
\end{abstract}

\section{Introduction}

The statistical analysis and modeling of lifetime data are crucial in almost all areas of knowledge including medical science, engineering, behavioral science, insurance and finance among others. The two important one parameter lifetime distributions for modeling lifetime data were exponential distribution and Lindley distribution, introduced by Lindley [1]. The Lindley distribution is defined by its probability density function (pdf) and cumulative distribution function (cdf)

$$
\begin{gathered}
f_{1}(x ; \theta)=\frac{\theta^{2}}{\theta+1}(1+x) e^{-\theta x} ; \quad x>0, \theta>0 \\
F_{1}(x ; \theta)=1-\left(1+\frac{\theta x}{\theta+1}\right) e^{-\theta x} ; \quad x>0, \theta>0
\end{gathered}
$$

where $\theta$ is a scale parameter. Ghitany et al [2] have discussed statistical properties, estimation of parameter and application of Lindley distribution. Shanker et al [3] have detailed critical study on applications of exponential and Lindley distributions for modeling lifetime data from biomedical science and engineering and observed that in majority of datasets these two distributions are not suitable. In search for a new lifetime distribution which gives a better fit than both exponential and Lindley distributions, Shanker [4] proposed Sujatha distribution defined by its pdf and cdf

$$
\begin{gathered}
f_{2}(x ; \theta)=\frac{\theta^{3}}{\theta^{2}+\theta+2}\left(1+x+x^{2}\right) e^{-\theta x} ; x>0, \theta>0 \\
F_{2}(x ; \theta)=1-\left[1+\frac{\theta x(\theta x+\theta+2)}{\theta^{2}+\theta+2}\right] e^{-\theta x} ; x>0, \theta>0
\end{gathered}
$$


where $\theta$ is a scale parameter. It should be noted that Lindley distribution is a convex combination of exponential $(\theta)$ and a gamma $(2, \theta)$ distributions whereas Sujatha distribution is a convex combination of exponential $(\theta)$, a gamma $(2, \theta)$ and a gamma $(3, \theta)$ distributions.

The first four moments about origin and central moments of Sujatha distribution obtained by Shanker [4] are

$$
\begin{gathered}
\mu_{1}^{\prime}=\frac{\theta^{2}+2 \theta+6}{\theta\left(\theta^{2}+\theta+2\right)} \quad \mu_{2}^{\prime}=\frac{2\left(\theta^{2}+3 \theta+12\right)}{\theta^{2}\left(\theta^{2}+\theta+2\right)} \quad \mu_{3}^{\prime}=\frac{6\left(\theta^{2}+4 \theta+20\right)}{\theta^{3}\left(\theta^{2}+\theta+2\right)} \quad \mu_{4}^{\prime}=\frac{24\left(\theta^{2}+5 \theta+30\right)}{\theta^{4}\left(\theta^{2}+\theta+2\right)} \\
\mu_{2}=\frac{\theta^{4}+4 \theta^{3}+18 \theta^{2}+12 \theta+12}{\theta^{2}\left(\theta^{2}+\theta+2\right)^{2}} \\
\mu_{3}=\frac{2\left(\theta^{6}+6 \theta^{5}+36 \theta^{4}+44 \theta^{3}+54 \theta^{2}+36 \theta+24\right)}{\theta^{3}\left(\theta^{2}+\theta+2\right)^{3}} \\
\mu_{4}=\frac{3\left(3 \theta^{8}+24 \theta^{5}+172 \theta^{6}+376 \theta^{5}+736 \theta^{4}+864 \theta^{3}+912 \theta^{2}+480 \theta+240\right)}{\theta^{4}\left(\theta^{2}+\theta+2\right)^{4}}
\end{gathered}
$$

Shanker [4] has discussed its important properties including shapes of density function for varying values of parameter, hazard rate function, mean residual life function, stochastic ordering, mean deviations, Bonferroni and Lorenz curves, and stress-strength reliability. Shanker [4] discussed the maximum likelihood estimation of parameter and showed the applications of Sujatha distribution to model lifetime data from biomedical science and engineering. Shanker [5] has introduced Poisson- Sujatha distribution (PSD), a Poisson mixture of Sujatha distribution, and studied its properties, estimation of parameter and applications to model count data. Shanker and Hagos [6] have discussed zero-truncated Poisson- Sujatha distribution (ZTPSD) and applications for modeling count data excluding zero counts. Shanker and Hagos [7] have also studied size-biased Poisson- Sujatha distribution and its applications for count data excluding zero counts.

Shanker [8] proposed a two-parameter quasi Sujatha distribution (QSD), which includes Sujatha distribution and size-biased Lindley distribution (SBLD) as particular cases for $\alpha=\theta$ and $\alpha=0$, respectively and defined by its pdf and cdf

$$
\begin{gathered}
f_{3}(x ; \theta, \alpha)=\frac{\theta^{2}}{\alpha \theta+\theta+2}\left(\alpha+\theta x+\theta x^{2}\right) e^{-\theta x} ; \quad x>0, \theta>0, \alpha \geq 0 \\
F_{3}(x ; \theta, \alpha)=1-\left[1+\frac{\theta x(\theta x+\theta+2)}{\alpha \theta+\theta+2}\right] e^{-\theta x} ; x>0, \theta>0, \alpha \geq 0 .
\end{gathered}
$$

Statistical properties including moments based measures, hazard rate function, mean residual life function, stochastic ordering, mean deviation, Bonferroni and Lorenz curves, stress-strength reliability along with estimation of parameters using both the method of moments and the method of maximum likelihood and applications of QSD have been discussed by Shanker [8]. It has been established through a real lifetime data that QSD gives much closer fit over one parameter exponential, Lindley and Sujatha distributions and two-parameter lognormal, gamma and Weibull distributions.

Recently, Mussie and Shanker [9] proposed a two-parameter Sujatha distribution (TPSD) defined by its pdf and cdf

$$
\begin{aligned}
& f_{4}(x ; \theta, \alpha)=\frac{\theta^{3}}{\alpha \theta^{2}+\theta+2}\left(\alpha+x+x^{2}\right) e^{-\theta x} ; \quad x>0, \theta>0, \alpha \geq 0 \\
& F_{4}(x ; \theta, \alpha)=1-\left[1+\frac{\theta x(\theta x+\theta+2)}{\alpha \theta^{2}+\theta+2}\right] e^{-\theta x} ; \quad x>0, \theta>0, \alpha \geq 0 .
\end{aligned}
$$

Like QSD, TPSD also includes Sujatha distribution and SBLD as particular cases for $\alpha=1$ and $\alpha=0$, respectively.

Statistical properties including coefficient of variation, skewness, kurtosis, index of dispersion, hazard rate function, mean residual life function, stochastic ordering, mean deviations, Bonferroni and Lorenz curves, stress-strength reliability along with estimation of parameters using both the method of moments and the method of maximum likelihood and applications of TPSD have been discussed in Mussie and Shanker [9]. In search for a new two-parameter Sujatha distribution (NTPSD), Mussie and Shanker [10] proposed the following NTPSD defined by its pdf and cdf

$$
\begin{aligned}
& f_{5}(x ; \theta, \alpha)=\frac{\theta^{3}}{\theta^{2}+\alpha \theta+2}\left(1+\alpha x+x^{2}\right) e^{-\theta x} ; \quad x>0, \theta>0, \alpha \geq 0 \\
& F_{5}(x ; \theta, \alpha)=1-\left[1+\frac{\theta x(\theta x+\alpha \theta+2)}{\theta^{2}+\alpha \theta+2}\right] e^{-\theta x}, \quad x>0, \theta>0, \alpha \geq 0 .
\end{aligned}
$$


Mussie and Shanker [10] have discussed statistical properties including coefficient of variation, skewness, kurtosis, index of dispersion, hazard rate function, mean residual life function, stochastic ordering, mean deviations, Bonferroni and Lorenz curves, stress-strength reliability, and estimation of the parameters using maximum likelihood estimation. Some numerical examples have also been presented by Mussie and Shanker [10] to test the goodness of fit of NTPSD over one parameter exponential, Lindley and Sujatha distributions and two-parameter Sujatha distribution (TPSD).

Note that NTPSD includes Sujatha distribution and Akash distribution proposed by Shanker [11] as particular cases for $\alpha=1$ and $\alpha=0$, respectively.

In this paper another two-parameter Sujatha distribution (ATPSD) which includes both exponential distribution and Sujatha distribution as particular cases has been suggested. Its important properties including hazard rate function, mean residual life function, stochastic ordering, mean deviations, Bonferroni and Lorenz curves, stress-strength reliability have been discussed. The estimation of the parameters has been discussed using maximum likelihood estimation. Two numerical examples have been given to test the goodness of fit of ATPSD and the fit has been compared with two-parameter QSD, TPSD and NTPSD and one parameter exponential, Lindley and Sujatha distributions.

\section{Another two-parameter Sujatha distribution}

Another two-parameter Sujatha distribution (ATPSD) having parameters $\theta$ and $\alpha$ is defined by its pdf.

$$
f_{6}(x ; \theta, \alpha)=\frac{\theta^{3}}{\theta^{2}+\alpha \theta+2 \alpha}\left(1+\alpha x+\alpha x^{2}\right) e^{-\theta x} ; \quad x>0, \theta>0, \alpha \geq 0,
$$

where $\theta$ is a scale parameter and $\alpha$ is a shape parameter. It can easily be verified that (2.1) reduces to Sujatha distribution (1.1), introduced by Shanker [4] and exponential distribution for $\alpha=1$ and $\alpha=0$, respectively.

Like Sujatha distribution, ATPSD (2.1) is also a three-component mixture of exponential $(\theta)$, gamma $(2, \theta)$ and gamma $(3, \theta)$ distributions. We have

$$
f_{6}(x ; \theta, \alpha)=p_{1} g_{1}(x ; \theta)+p_{2} g_{2}(x ; \theta, 2)+\left(1-p_{1}-p_{2}\right) g_{3}(x ; \theta, 3)
$$

where

$$
\begin{gathered}
p_{1}=\frac{\theta^{2}}{\theta^{2}+\alpha \theta+2 \alpha}, \quad p_{2}=\frac{\alpha \theta}{\theta^{2}+\alpha \theta+2 \alpha}, \quad g_{1}(x ; \theta)=\theta e^{-\theta x} ; \quad x>0, \theta>0 \\
g_{2}(x ; \theta, 2)=\frac{\theta^{2}}{\Gamma(2)} e^{-\theta x} x^{2-1} ; \quad x>0, \theta>0, \quad g_{3}(x ; \theta, 3)=\frac{\theta^{3}}{\Gamma(3)} e^{-\theta x} x^{3-1} ; \quad x>0, \theta>0 .
\end{gathered}
$$

The corresponding cdf of ATPSD (2.1) can be obtained as

$$
F_{6}(x ; \theta, \alpha)=1-\left[1+\frac{\alpha \theta x(\theta x+\alpha \theta+2)}{\theta^{2}+\alpha \theta+2 \alpha}\right] e^{-\theta x} ; \quad x>0, \theta>0, \alpha \geq 0 .
$$

Behaviors of the pdf and the cdf of ATPSD for varying values $\theta$ of $\alpha$ the parameters and are shown in Figure 2.1 and Figure 2.2, respectively.

\section{Moments and related measures}

The moment generating function of ATPSD (2.1) can be obtained as

$$
\begin{aligned}
M_{X}(t) & =\frac{\theta^{3}}{\theta^{2}+\alpha \theta+2 \alpha} \int_{0}^{\infty} e^{-(\theta-t) x}\left(1+\alpha x+\alpha x^{2}\right) d x \\
& =\frac{\theta^{3}}{\theta^{2}+\alpha \theta+2 \alpha}\left[\frac{1}{(\theta-t)}+\frac{\alpha}{(\theta-t)^{2}}+\frac{2 \alpha}{(\theta-t)^{3}}\right] \\
& =\frac{\theta^{3}}{\theta^{2}+\alpha \theta+2 \alpha}\left[\frac{1}{\theta} \sum_{k=0}^{\infty}\left(\frac{t}{\theta}\right)^{k}+\frac{\alpha}{\theta^{2}} \sum_{k=0}^{\infty}\left(\begin{array}{c}
k+1 \\
k
\end{array}\right)\left(\frac{t}{\theta}\right)^{k}+\frac{2 \alpha}{\theta^{3}} \sum_{k=0}^{\infty}\left(\begin{array}{c}
k+2 \\
k
\end{array}\right)\left(\frac{t}{\theta}\right)^{k}\right] \\
& =\sum_{k=0}^{\infty} \frac{\theta^{2}+\alpha \theta(k+1)+\alpha(k+1)(k+2)}{\theta^{2}+\alpha \theta+2 \alpha}\left(\frac{t}{\theta}\right)^{k} .
\end{aligned}
$$

Thus, the $r^{t h}$ moment about origin of ATPSD (2.1), obtained as the coefficient of $\frac{t^{r}}{r !}$ in $M_{X}(t)$, is given by

$$
\mu_{r}^{\prime}=\frac{r !\left\{\theta^{2}+(r+1) \alpha \theta+(r+1)(r+2) \alpha\right\}}{\theta^{r}\left(\theta^{2}+\alpha \theta+2 \alpha\right)} ; \quad r=1,2,3, \ldots
$$



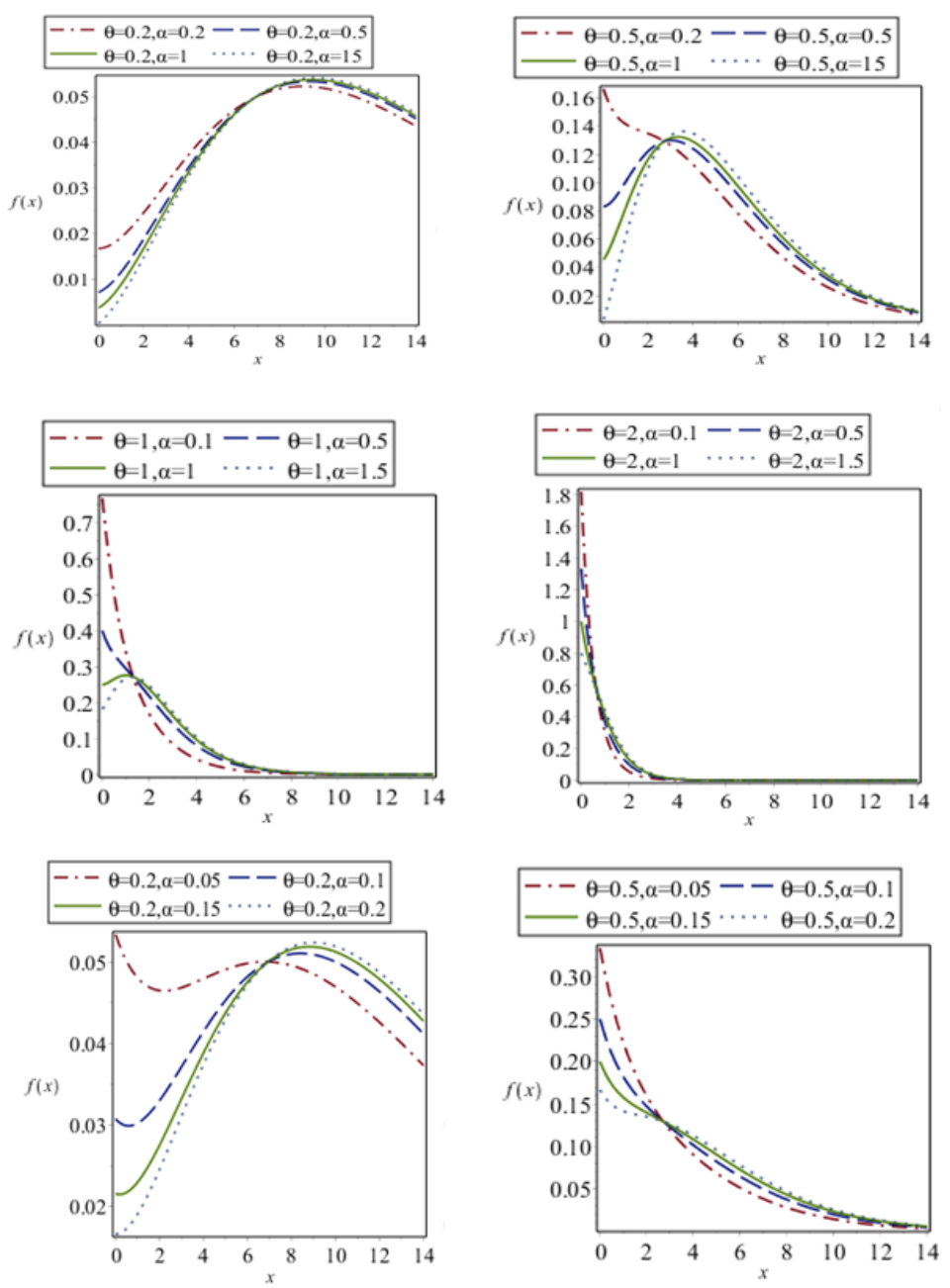

Figure 2.1: Behavior of the pdf of ATPSD for varying values of the parameters $\theta$ and $\alpha$

The first four moments about origin of ATPSD are obtained as

$$
\begin{gathered}
\mu_{1}^{\prime}=\frac{\theta^{2}+2 \alpha \theta+6 \alpha}{\theta\left(\theta^{2}+\alpha \theta+2 \alpha\right)}, \quad \mu_{2}^{\prime}=\frac{2\left(\theta^{2}+3 \alpha \theta+12 \alpha\right)}{\theta^{2}\left(\theta^{2}+\alpha \theta+2 \alpha\right)}, \\
\mu_{3}^{\prime}=\frac{6\left(\theta^{2}+4 \alpha \theta+20 \alpha\right)}{\theta^{3}\left(\theta^{2}+\alpha \theta+2 \alpha\right)}, \quad \mu_{4}^{\prime}=\frac{24\left(\theta^{2}+5 \alpha \theta+30 \alpha\right)}{\theta^{4}\left(\theta^{2}+\alpha \theta+2 \alpha\right)} .
\end{gathered}
$$

Using the relationship between moments about the mean and moments about the origin, the moments about mean of ATPSD are obtained as

$$
\begin{gathered}
\mu_{2}=\frac{2 \alpha^{2} \theta^{2}+4 \alpha \theta^{3}+\theta^{4}+12 \alpha^{2} \theta+16 \alpha \theta^{2}+12 \alpha^{2}}{\theta^{2}\left(\theta^{2}+\alpha \theta+2 \alpha\right)^{2}} \\
\mu_{3}=\frac{2\left(2 \alpha^{3} \theta^{3}+6 \alpha^{2} \theta^{4}+6 \alpha \theta^{5}+\theta^{6}+18 \alpha^{3} \theta^{2}+42 \alpha^{2} \theta^{3}+30 \alpha \theta^{4}+36 \alpha^{3} \theta+36 \alpha^{2} \theta^{2}+24 \alpha^{3}\right)}{\theta^{3}\left(\theta^{2}+\alpha \theta+2 \alpha\right)^{3}} \\
\mu_{4}=\frac{3\left(\begin{array}{c}
8 \alpha^{4} \theta^{4}+32 \alpha^{3} \theta^{5}+44 \alpha^{2} \theta^{6}+24 \alpha \theta^{7}+3 \theta^{8}+96 \alpha^{4} \theta^{3}+320 \alpha^{3} \theta^{4}+344 \alpha^{2} \theta^{5} \\
+128 \alpha \theta^{6}+336 \alpha^{4} \theta^{2}+768 \alpha^{3} \theta^{3}+408 \alpha^{2} \theta^{4}+480 \alpha^{4} \theta+576 \alpha^{3} \theta^{2}+240 \alpha^{4}
\end{array}\right)}{\theta^{4}\left(\theta^{2}+\alpha \theta+2 \alpha\right)^{4}}
\end{gathered}
$$

The coefficient of variation $(\mathrm{C} . \mathrm{V})$, coefficient of skewness $\left(\sqrt{\beta_{1}}\right)$, coefficient of kurtosis $\left(\beta_{2}\right)$ and index of dispersion $(\gamma)$ of ATPSD are given by

$$
C V=\frac{\sigma}{\mu_{1}}=\frac{\sqrt{2 \alpha^{2} \theta^{2}+4 \alpha \theta^{3}+\theta^{4}+12 \alpha^{2} \theta}+16 \alpha \theta^{2}+12 \alpha^{2}}{\theta^{2}+2 \alpha \theta+6 \alpha}
$$



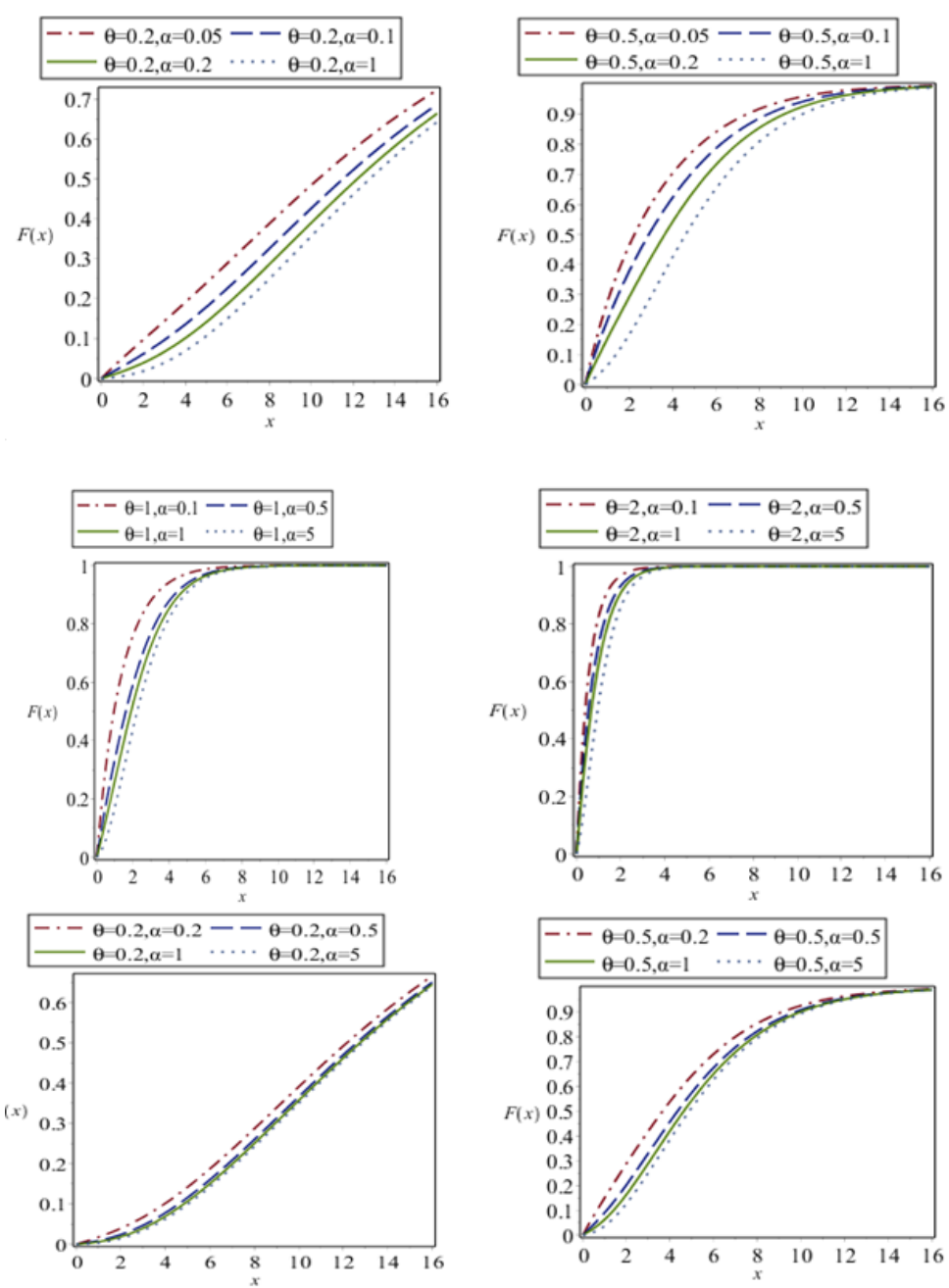

Figure 2.2: Behavior of the cdf of ATPSD for varying values of the parameters $\theta$ and $\alpha$

$$
\begin{gathered}
\sqrt{\beta_{1}}=\frac{\mu_{3}}{\mu_{2}^{3 / 2}}=\frac{2\left(2 \alpha^{3} \theta^{3}+6 \alpha^{2} \theta^{4}+6 \alpha \theta^{5}+\theta^{6}+18 \alpha^{3} \theta^{2}+42 \alpha^{2} \theta^{3}+30 \alpha \theta^{4}+36 \alpha^{3} \theta+36 \alpha^{2} \theta^{2}+24 \alpha^{3}\right)}{\left(2 \alpha^{2} \theta^{2}+4 \alpha \theta^{3}+\theta^{4}+12 \alpha^{2} \theta+16 \alpha \theta^{2}+12 \alpha^{2}\right)^{3 / 2}} \\
\beta_{2}=\frac{\mu_{4}}{\mu_{2}^{2}}=\mu_{4}=\frac{3\left(\begin{array}{c}
8 \alpha^{4} \theta^{4}+32 \alpha^{3} \theta^{5}+44 \alpha^{2} \theta^{6}+24 \alpha \theta^{7}+3 \theta^{8}+96 \alpha^{4} \theta^{3}+320 \alpha^{3} \theta^{4}+344 \alpha^{2} \theta^{5} \\
+128 \alpha \theta^{6}+336 \alpha^{4} \theta^{2}+768 \alpha^{3} \theta^{3}+408 \alpha^{2} \theta^{4}+480 \alpha^{4} \theta+576 \alpha^{3} \theta^{2}+240 \alpha^{4}
\end{array}\right)}{\left(2 \alpha^{2} \theta^{2}+4 \alpha \theta^{3}+\theta^{4}+12 \alpha^{2} \theta+16 \alpha \theta^{2}+12 \alpha^{2}\right)^{2}} \\
\gamma=\frac{\sigma^{2}}{\mu_{1}^{\prime}}=\frac{2 \alpha^{2} \theta^{2}+4 \alpha \theta^{3}+\theta^{4}+12 \alpha^{2} \theta+16 \alpha \theta^{2}+12 \alpha^{2}}{\theta\left(\theta^{2}+\alpha \theta+2 \alpha\right)\left(\theta^{2}+2 \alpha \theta+6 \alpha\right)}
\end{gathered}
$$

It can be easily verified that these statistical constants of ATPSD reduce to the corresponding statistical constants of Sujatha and exponential distributions at $\alpha=1$ and $\alpha=0$ respectively.

The behaviors of C.V., $\sqrt{\beta_{1}}, \beta_{2}$ and $\gamma$, for varying values of the parameters $\theta$ and $\alpha$ have been shown numerically in Tables $1,2,3$ and 4 . For a given value of $\alpha, \mathrm{C}$.V. increases as the value of $\theta$ increases. But for values $1 \leq \theta \leq 5, \mathrm{C}$.V. decreases as the value of $\alpha$ increases.

Since $\sqrt{\beta_{1}}>0$, ATPSD is always positively skewed, and this means that ATPSD is a suitable model for positively skewed lifetime data.

Since $\beta_{2}>3$, ATPSD is always leptokurtic, which means that ATPSD is more peaked than the normal curve, thus ATPSD is suitable for lifetime data which are leptokurtic.

Index of dispersion of ATPSD for varying values of parameters and .

As long as $0 \leq \theta \leq 1$ and $0 \leq \alpha \leq 5$, the nature of ATPSD is over dispersed $\left(\sigma^{2}>\mu_{1}^{\prime}\right)$ and for $1 \leq \theta \leq 5$ and $0 \leq \alpha \leq 5$, the nature of ATPSD is under dispersed $\left(\sigma^{2}<\mu_{1}^{\prime}\right)$. 
Table 1: C.V. of ATPSD for varying values of parameters $\theta$ and $\alpha$.

\begin{tabular}{|l|l|l|l|l|l|l|l|}
\hline$\alpha / \theta$ & 0.2 & 0.5 & 1 & 2 & 3 & 4 & 5 \\
\hline 0.2 & 0.640678 & 0.790787 & 0.945247 & 1.013246 & 1.015576 & 1.012149 & 1.009163 \\
\hline 0.5 & 0.614004 & 0.702377 & 0.836660 & 0.962250 & 0.99661 & 1.005655 & 1.007547 \\
\hline 1 & 0.604466 & 0.662392 & 0.761739 & 0.892143 & 0.951190 & 0.977525 & 0.989835 \\
\hline 2 & 0.599565 & 0.639569 & 0.708329 & 0.816497 & 0.882958 & 0.922627 & 0.946881 \\
\hline 3 & 0.597911 & 0631470 & 0.687023 & 0.778162 & 0.840635 & 0.882516 & 0.911036 \\
\hline 4 & 0.597080 & 0.627321 & 0.675561 & 0.755148 & 0.812529 & 0.853461 & 0.883077 \\
\hline 5 & 0.596580 & 0.624798 & 0.668399 & 0.739814 & 0.792609 & 0.831700 & 0.861102 \\
\hline
\end{tabular}

Table 2: Coefficient skewness $\left(\sqrt{\beta_{1}}\right)$ of ATPSD for varying values of parameters $\theta$ and $\alpha$

\begin{tabular}{|l|l|l|l|l|l|l|l|}
\hline$\alpha / \theta$ & 0.2 & 0.5 & 1 & 2 & 3 & 4 & 5 \\
\hline 0.2 & 1.114210 & 1.248863 & 1.643745 & 1.984109 & 2.045477 & 2.048672 & 2.041683 \\
\hline 0.5 & 1.133836 & 1.154381 & 1.365976 & 1.745907 & 1.916915 & 1.984875 & 2.011218 \\
\hline 1 & 1.145006 & 1.145839 & 1.247611 & 1.535588 & 1.733747 & 1.848046 & 1.912879 \\
\hline 2 & 1.151691 & 1.153577 & 1.201582 & 1.377838 & 1.541364 & 1.662624 & 1.748583 \\
\hline 3 & 1.154099 & 1.158904 & 1.194050 & 1.320932 & 1.451434 & 1.558992 & 1.642899 \\
\hline 4 & 1.155338 & 1.162202 & 1.192975 & 1.294584 & 1.402342 & 1.496069 & 1.573228 \\
\hline 5 & 1.156092 & 1.164402 & 1.193449 & 1.280549 & 1.372667 & 1.455018 & 1.525066 \\
\hline
\end{tabular}

Table 3: Coefficient of kurtosis $\left(\beta_{2}\right)$ of ATPSD for varying values of parameters $\theta$ and $\alpha$

\begin{tabular}{|l|l|l|l|l|l|l|l|}
\hline$\alpha / \theta$ & 0.2 & 0.5 & 1 & 2 & 3 & 4 & 5 \\
\hline 0.2 & 4.869916 & 5.109996 & 6.633262 & 8.690336 & 9.230612 & 9.313262 & 9.289003 \\
\hline 0.5 & 4.941280 & 4.924032 & 5.510204 & 7.214400 & 8.270528 & 8.774988 & 9.001011 \\
\hline 1 & 4.973635 & 4.944566 & 5.170213 & 6.214990 & 7.193906 & 7.868405 & 8.297711 \\
\hline 2 & 4.991667 & 4.984856 & 5.082378 & 5.625000 & 6.285420 & 6.865586 & 7.326691 \\
\hline 3 & 4.997968 & 5.004164 & 5.080437 & 5.451135 & 5.931292 & 6.392963 & 6.794445 \\
\hline 4 & 5.001174 & 5.015076 & 5.087074 & 5.380592 & 5.758343 & 6.135306 & 6.478444 \\
\hline 5 & 5.003116 & 5.022048 & 5.093943 & 5.346882 & 5.661781 & 5.979645 & 6.275987 \\
\hline
\end{tabular}

Table 4: Index of dispersion $(\gamma)$ of ATPSD for varying values of parameters $\theta$ and $\alpha$

\begin{tabular}{|l|l|l|l|l|l|l|l|}
\hline$\alpha / \theta$ & 0.2 & 0.5 & 1 & 2 & 3 & 4 & 5 \\
\hline 0.2 & .5643939 & 2.751515 & 1.451923 & 0.641667 & 0.391930 & 0.279936 & 0.217569 \\
\hline 0.5 & 5.357375 & 2.466667 & 1.400000 & 0.694444 & 0.431884 & 0.306064 & 0.235088 \\
\hline 1 & 5.252329 & 2.313480 & 1.305556 & 0.696429 & 0.452381 & 0.325758 & 0.251067 \\
\hline 2 & 5.197861 & 2.220551 & 1.218487 & 0.666667 & 0.451356 & 0.334416 & 0.262078 \\
\hline 3 & 5.179408 & 2.186717 & 1.180000 & 0.643382 & 0.441667 & 0.332150 & 0.263431 \\
\hline 4 & 5.170124 & 2.169221 & 1.158508 & 0.627273 & 0.432547 & 0.327778 & 0.261904 \\
\hline 5 & 5.164536 & 2.158531 & 1.144817 & 0.615741 & 0.424979 & 0.323306 & 0.259524 \\
\hline
\end{tabular}




\section{Reliability properties}

In this section, reliability properties of ATPSD including hazard rate function, mean residual life function, stochastic ordering and stressstrength reliability have been discussed.

\subsection{Hazard rate function and mean residual life function}

Let $X$ be a continuous random variable with pdf $f(x)$ and cdf $F(x)$. The hazard rate function (also known as failure rate function), $h(x)$ and the mean residual function, $m(x)$ of $X$ are respectively defined as

$$
h(x)=\lim _{\Delta x \rightarrow 0} \frac{p(X<x+\Delta x \mid X>x)}{\Delta x}=\frac{f(x)}{1-F(x)}
$$

and

$$
m(x)=E[X-x \mid X>x]=\frac{1}{1-F(x)} \int_{x}^{\infty}[1-F(t)] d t
$$

The corresponding hazard rate function, $h(x)$ and the mean residual function $m(x)$ of ATPSD (2.1) are thus obtained as

$$
h(x)=\frac{\theta^{3}\left(1+\alpha x+\alpha x^{2}\right)}{\theta^{2}\left(1+\alpha x+\alpha x^{2}\right)+\alpha(2 \theta x+\theta+2)}
$$

and

$m(x)=\frac{\theta^{2}+\alpha \theta+2 \alpha}{\left[\left(\theta^{2}+\alpha \theta+2 \alpha\right)+\alpha \theta x(\theta x+\theta+2)\right] e^{-\theta x}} \int_{x}^{\infty}\left[1+\frac{\alpha \theta t(\theta t+\theta+2)}{\theta^{2}+\alpha \theta+2 \alpha}\right] e^{-\theta t} d t=\frac{\alpha \theta^{2} x^{2}+\alpha \theta(\theta+4) x+\left(\theta^{2}+2 \alpha \theta+6 \alpha\right)}{\theta\left[\alpha \theta^{2} x^{2}+\alpha \theta(\theta+2) x+\left(\theta^{2}+\alpha \theta+2 \alpha\right)\right]}$

It can be easily verified that $h(0)=\frac{\theta^{3}}{\theta^{2}+\alpha \theta+2 \alpha}=f(0)$ and $m(0)=\frac{\theta^{2}+2 \alpha \theta+6 \alpha}{\theta\left(\theta^{2}+\alpha \theta+2 \alpha\right)}=\mu_{1}^{\prime}$

It can also be easily verified that these expressions for $h(x)$ and $m(x)$ of ATPSD reduce to the corresponding $h(x)$ and $m(x)$ of Sujatha distribution ar $\alpha=1$.

The behavior of $h(x)$ and $m(x)$ of ATPSD for different values of its parameters $\theta$ and $\alpha$ are shown in Figures 4.1 and 4.2, respectively. It is obvious from the graphs of $h(x)$ that it takes different shapes including monotonically increasing, upside bathtub and downside bathtub, etc., whereas $m(x)$ is monotonically decreasing function.

\subsection{Stochastic ordering}

Stochastic ordering of positive continuous random variable is an important tool for judging the comparative behavior of continuous distributions. A random variable $X$ is said to be smaller than a random variable $Y$ in the
i. stochastic order $\left(X \leq_{s t} Y\right)$ if $F_{x}(x) \geq F_{y}(x)$ for all $x$,
ii. hazard rate order $\left(X \leq_{h t} Y\right)$ if $h_{x}(x) \geq h_{y}(x)$ for all $x$,
iii. mean residual life order $\left(X \leq_{m r l} Y\right)$ if $m_{x}(x) \leq m_{y}(x)$ for all $x$,
iv. likelihood ratio order $\left(X \leq_{l r} Y\right)$ if $\frac{f_{x}(x)}{f_{y}(x)}$ decreases in $x$.

The following results due to Shaked and Shanthikumar [12] are well known for establishing stochastic ordering of distributions

$$
\begin{gathered}
X \leq_{l r} Y \Rightarrow X \leq_{h r} Y \Rightarrow X \leq_{m l r} Y \\
\Downarrow_{x \leq_{s t} y}
\end{gathered}
$$

The ATPSD (2.1) is ordered with respect to the strongest "likelihood ratio" ordering as established in the following theorem:

Theorem 4.1. Let $X \sim \operatorname{ATPSD}\left(\theta_{1}, \alpha_{1}\right)$ and $Y \sim A T P S D\left(\theta_{2}, \alpha_{2}\right)$. If $\theta_{1}>\theta_{2}$ and $\alpha_{1}=\alpha_{2}$ or $\theta_{1}=\theta_{2}$ and $\alpha_{1}<\alpha_{2}$, then $X \leq{ }_{l r} Y$ and hence $X \leq_{h r} Y, X \leq_{m r l} Y$ and $X \leq_{s t} Y$.

Proof. We have

$$
\begin{gathered}
\frac{f_{x}\left(x ; \theta_{1}, \alpha_{1}\right)}{f_{y}\left(x ; \theta_{2}, \alpha_{2}\right)}=\frac{\theta_{1}^{3}\left(\theta_{2}^{2}+\alpha_{2} \theta_{2}+2 \alpha_{2}\right)}{\theta_{2}^{3}\left(\theta_{1}^{2}+\alpha_{1} \theta_{1}+2 \alpha_{1}\right)}\left(\frac{1+\alpha_{1} x+\alpha_{1} x^{2}}{1+\alpha_{2} x+\alpha_{2} x^{2}}\right) e^{-\left(\theta_{1}-\theta_{2}\right) x ; \quad x>0} \\
\ln \frac{f_{x}\left(x ; \theta_{1}, \alpha_{1}\right)}{f_{y}\left(x ; \theta_{2}, \alpha_{2}\right)}=\ln \left[\frac{\theta_{1}^{3}\left(\theta_{2}^{2}+\alpha_{2} \theta_{2}+2 \alpha_{2}\right)}{\theta_{2}^{3}\left(\theta_{1}^{2}+\alpha_{1} \theta_{1}+2 \alpha_{1}\right)}\right]+\ln \left(\frac{1+\alpha_{1} x+\alpha_{1} x^{2}}{1+\alpha_{2} x+\alpha_{2} x^{2}}\right)-\left(\theta_{1}-\theta_{2}\right) x ; \quad x>0
\end{gathered}
$$



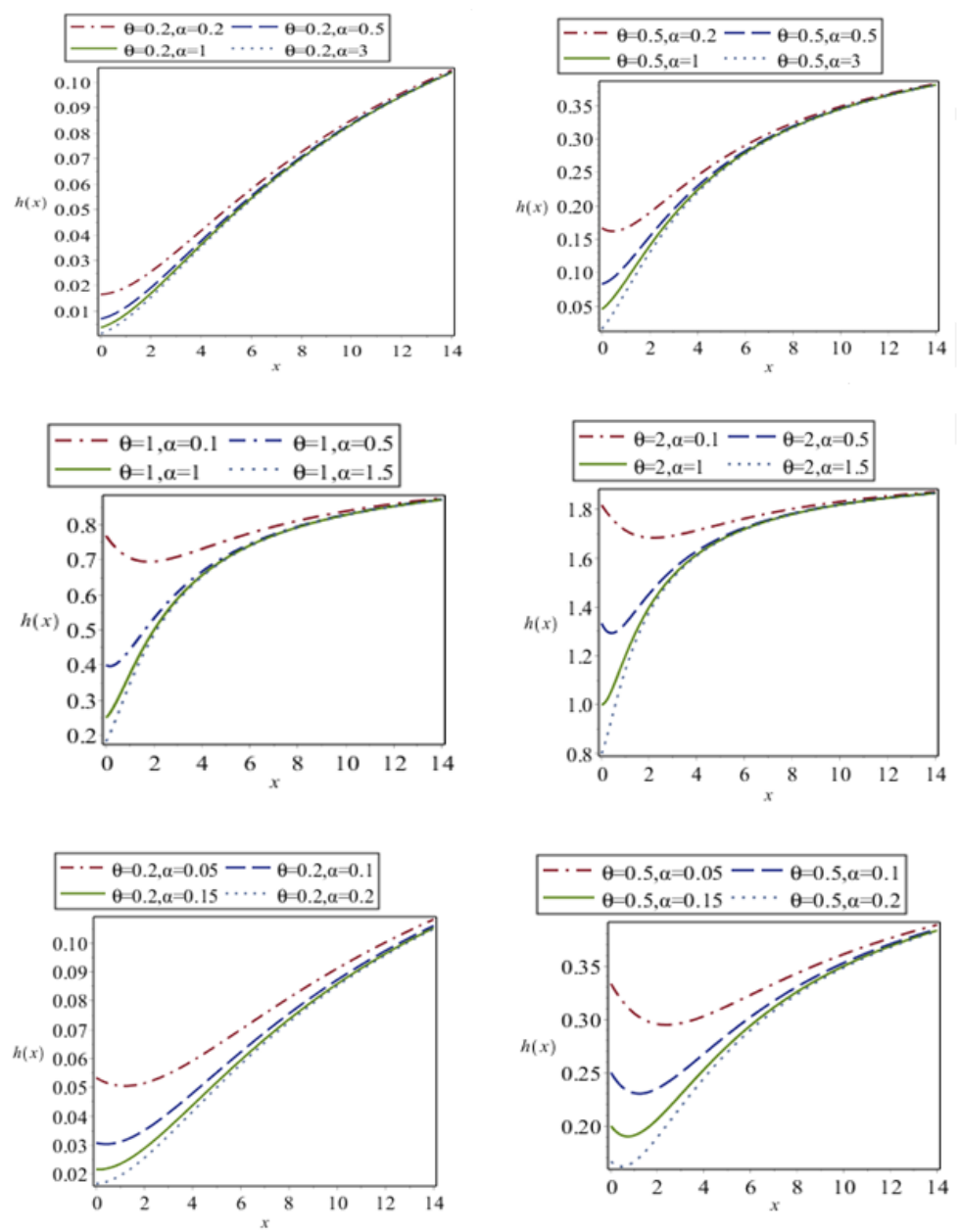

Figure 4.1: Behavior of the $h(x)$ of ATPSD for selected values of the parameters $\theta$ and $\alpha$

This gives

$$
\frac{d}{d x} \ln \frac{f_{x}\left(x ; \theta_{1}, \alpha_{1}\right)}{f_{y}\left(x ; \theta_{2}, \alpha_{2}\right)}=\frac{\left(\alpha_{1}-\alpha_{2}\right)-\left(\alpha_{1}-\alpha_{2}\right) x^{2}}{\left(1+\alpha_{1} x+x^{2}\right)\left(1+\alpha_{2} x+x^{2}\right)}-\left(\theta_{1}-\theta_{2}\right) .
$$

Thus for $\left(\theta_{1}>\theta_{2}\right.$ and $\left.\alpha_{1}=\alpha_{2}\right)$ or $\left(\theta_{1}=\theta_{2}\right.$ and $\left.\alpha_{1}<\alpha_{2}\right), \frac{d}{d x} \ln \frac{f_{x}\left(x ; \theta_{1}, \alpha_{1}\right)}{f_{y}\left(x ; \theta_{2}, \alpha_{2}\right)}<0$. This means that $X \leq_{l r} Y$ and hence $X \leq{ }_{h r} Y, X \leq{ }_{m r l} Y$ and $X \leq_{s t} Y$. This shows flexibility of ATPSD over Sujatha distribution.

\subsection{Stress-strength reliability}

The stress-strength reliability of a component illustrates the life of the component which has random strength $X$ that is subjected to random stress $Y$. When the stress of the component $Y$ applied to it exceeds the strength of the component $X$, the component fails instantly and the component will function satisfactorily till $X>Y$. Therefore, $R=P(Y<X)$ is a measure of the component reliability and is known as stress-strength reliability in statistical literature. It has extensive application in almost all areas of knowledge especially in engineering such as structure, deterioration of rocket motor, static fatigue of ceramic component, aging of concrete pressure vessels etc.

Let $X$ and $Y$ be independent strength and stress random variables having ATPSD (2.1) with parameter $\left(\theta_{1}, \alpha_{1}\right)$ and $\left(\theta_{2}, \alpha_{2}\right)$ respectively. Then the stress-strength reliability $R$ of ATPSD can be obtained as

$$
R=P(Y<X)=\int_{0}^{\infty} P(Y<X \mid X=x) f_{x}(x) d x=\int_{0}^{\infty} f_{6}\left(x ; \theta_{1}, \alpha_{1}\right) F_{6}\left(x ; \theta_{2}, \alpha_{2}\right) d x
$$



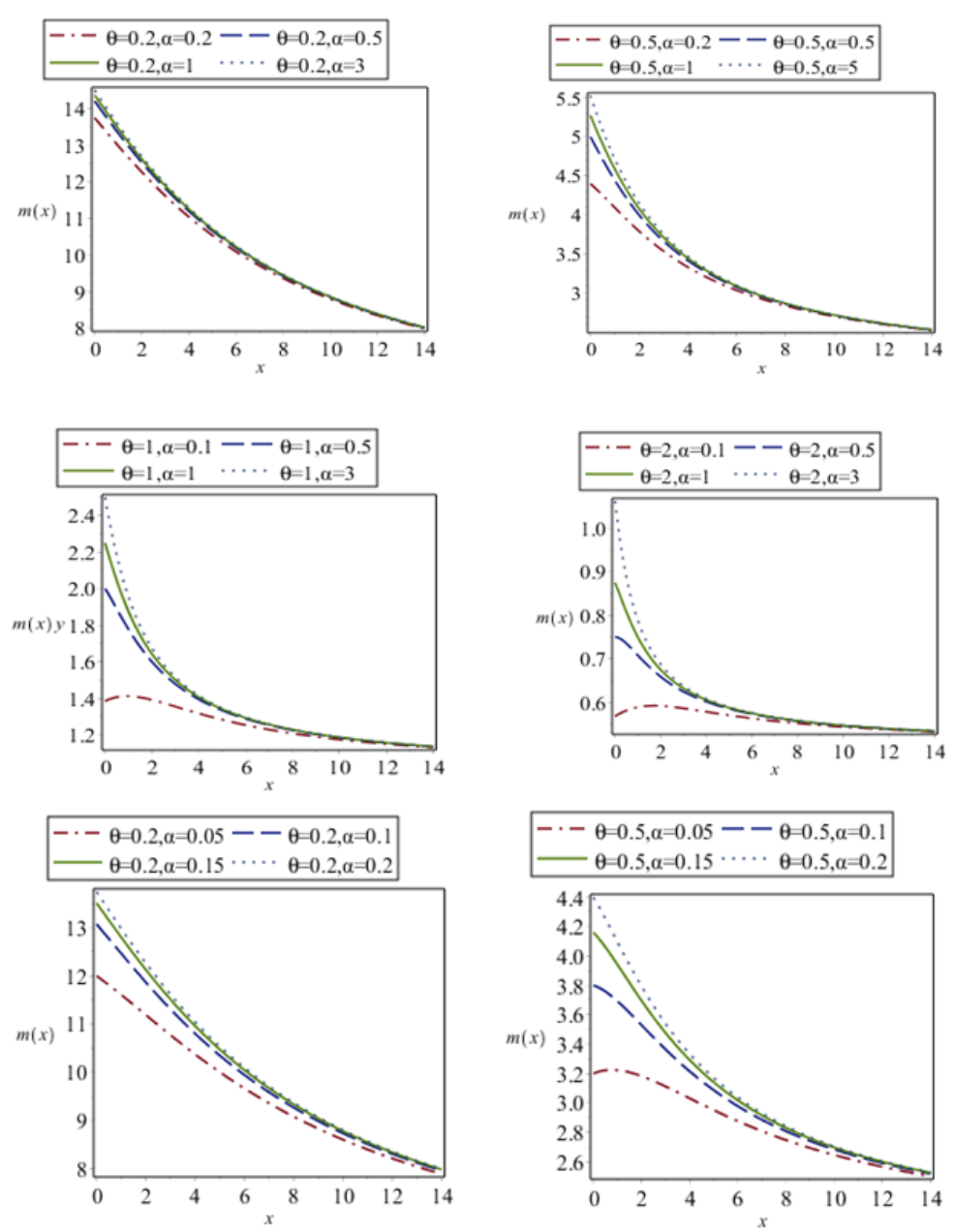

Figure 4.2: Behavior of the $m(x)$ of ATPSD for selected values of the parameters $\theta$ and $\alpha$

$$
\begin{aligned}
R & =1-\frac{\theta_{1}^{3}\left[\theta_{2}^{6}+\left(\alpha_{1}+2 \alpha_{2}+4 \theta_{1}\right) \theta_{2}^{5}+\left(3 \alpha_{1} \alpha_{2}+3 \alpha_{1} \theta_{1}+7 \alpha_{1} \alpha_{2} \theta_{1}+6 \theta_{1}^{2}+2 \alpha_{1}+6 \alpha_{2}\right) \theta_{2}^{4}\right]}{\left(\theta_{1}^{2}+\alpha_{1} \theta_{1}+2 \alpha_{1}\right)\left(\theta_{2}^{2}+\alpha_{2} \theta_{2}+2 \alpha_{2}\right)\left(\theta_{1}+\theta_{2}\right)^{5}} \\
& +\frac{\theta_{1}^{3}\left(7 \alpha_{1} \alpha_{2} \theta_{1}+3 \alpha_{1} \theta_{1}^{2}+9 \alpha_{2} \theta_{1}^{2}+4 \theta_{1}^{3}+20 \alpha_{1} \alpha_{2}+4 \alpha_{1} \theta_{1}+18 \alpha_{2} \theta_{1}\right) \theta_{2}^{3}}{\left(\theta_{1}^{2}+\alpha_{1} \theta_{1}+2 \alpha_{1}\right)\left(\theta_{2}^{2}+\alpha_{2} \theta_{2}+2 \alpha_{2}\right)\left(\theta_{1}+\theta_{2}\right)^{5}} \\
& +\frac{\theta_{1}^{3}\left(5 \alpha_{1} \alpha_{2} \theta_{1}^{2}+\alpha_{1} \theta_{1}^{3}+5 \alpha_{1} \alpha_{2} \theta_{1}^{3}+\theta_{1}^{4}+30 \alpha_{1} \alpha_{2} \theta_{1}+2 \alpha_{1} \theta_{1}^{2}+20 \alpha_{2} \theta_{1}^{2}+40 \alpha_{1} \alpha_{2}\right) \theta_{2}^{2}}{\left(\theta_{1}^{2}+\alpha_{1} \theta_{1}+2 \alpha_{1}\right)\left(\theta_{2}^{2}+\alpha_{2} \theta_{2}+2 \alpha_{2}\right)\left(\theta_{1}+\theta_{2}\right)^{5}} \\
& +\frac{\theta_{1}^{3}\left[\left(\alpha_{1} \alpha_{2} \theta_{1}^{2}+\alpha_{2} \theta_{1}^{3}+12 \alpha_{1} \alpha_{2} \theta_{1}+10 \alpha_{2} \theta_{1}^{2}+20 \alpha_{1} \alpha_{2}\right) \theta_{1} \theta_{2}+2\left(\theta_{1}^{2}+\theta_{1}+2\right) \alpha_{1} \theta_{1}^{2}\right]}{\left(\theta_{1}^{2}+\alpha_{1} \theta_{1}+2 \alpha_{1}\right)\left(\theta_{2}^{2}+\alpha_{2} \theta_{2}+2 \alpha_{2}\right)\left(\theta_{1}+\theta_{2}\right)^{5}}
\end{aligned}
$$

It can easily be verified that the stress-strength reliability of Sujatha distribution and exponential distribution are particular cases of stress-strength reliability of ATPSD at $\alpha_{1}=\alpha_{2}=1$, and $\alpha_{1}=\alpha_{2}=0$, respectively.

\section{Statistical properties}

\subsection{Mean deviations}

The amount of scatter in a population is evidently measured to some extent by the totality of deviations from the mean and the median. These are known as the mean deviation about the mean and the mean deviation about the median and are defined as

$$
\delta_{1}(X)=\int_{0}^{\infty}|x-\mu| f(x) d x \quad \delta_{2}(X)=\int_{0}^{\infty}|x-M| f(x) d x
$$

respectively., where $\mu=E(X)$ and $M=$ median $(X)$. The measures $\delta_{1}(X)$ and $\delta_{2}(X)$ can be calculated using the following relationships

$$
\begin{aligned}
\delta_{1}(X) & =\int_{0}^{\mu}(\mu-x) f(x) d x+\int_{\mu}^{\infty}(x-\mu) f(x) d x \\
& =\mu F(\mu)-\int_{0}^{\mu} f(x) d x-\mu[1-F(\mu)]+\int_{\mu}^{\infty} x f(x) d x=2 \mu F(\mu)-2 \mu+2 \int_{\mu}^{\infty} x f(x) d x=2 \mu F(\mu)-2 \int_{\mu}^{\infty} x f(x) d x
\end{aligned}
$$


and

$$
\begin{aligned}
\delta_{2}(X) & =\int_{0}^{M}(M-x) f(x) d x+\int_{M}^{\infty}(x-M) f(x) d x \\
& =M F(M)-\int_{0}^{M} f(x) d x-M[1-F(M)]+\int_{M}^{\infty} x f(x) d x=\mu+2 \int_{M}^{\infty} x f(x) d x=\mu-2 \int_{0}^{M} x f(x) d x
\end{aligned}
$$

Using the pdf (2.1) and expression for the mean of ATPSD, we get

$$
\begin{gathered}
\int_{0}^{\mu} x f_{6}(x)(x ; \theta, \alpha) d x=\mu-\frac{\left[\theta^{3}\left(\alpha \mu^{3}+\alpha \mu^{2}+\mu\right)+\theta^{2}\left(3 \alpha \mu^{2}+2 \alpha \mu+1\right)+2 \alpha \theta(3 \mu+1)+6 \alpha\right] e^{-\theta \mu}}{\theta\left(\theta^{2}+\alpha \theta+2 \alpha\right)} \\
\int_{0}^{M} x f_{6}(x)(x ; \theta, \alpha) d x=\mu-\frac{\left[\theta^{3}\left(\alpha M^{3}+\alpha M^{2}+M\right)+\theta^{2}\left(3 \alpha M^{2}+2 \alpha M+1\right)+2 \alpha \theta(3 M+1)+6 \alpha\right] e^{-\theta M}}{\theta\left(\theta^{2}+\alpha \theta+2 \alpha\right)}
\end{gathered}
$$

Using expressions from (5.1), (5.2), (5.3) and (5.4) and after some tedious algebraic simplifications, the mean deviation about the mean, $\delta_{1}(X)$ and the mean deviation about the median, $\delta_{2}(X)$ of ATPSD are obtained as

$$
\delta_{1}(X)=\frac{2\left[\theta^{3}\left(\alpha \mu^{2}+\alpha \mu+1\right)+2 \alpha \theta(2 \mu+1)+6 \alpha\right] e^{-\theta \mu}}{\theta\left(\theta^{2}+\alpha \theta+2 \alpha\right)}
$$

and

$$
\delta_{2}(X)=\frac{2\left[\theta^{3}\left(\alpha M^{2}+\alpha M+M\right)+\theta^{3}\left(3 \alpha M^{2}+2 \alpha M+1\right)+2 \alpha \theta(3 M+1)+6 \alpha\right] e^{-\theta M}}{\theta\left(\theta^{2}+\alpha \theta+2 \alpha\right)}-\mu
$$

\subsection{Bonferroni and Lorenz curves and indicies}

The Bonferroni and Lorenz curves, introduced by Bonferroni [13] and Bonferroni and Gini indices have applications not only in economics to study income and poverty, but also in other fields like reliability, demography and medical science. The Bonferroni and Lorenz curves are defined as

$$
B(p)=\frac{1}{p \mu} \int_{0}^{q} x f(x) d x=\frac{1}{p \mu}\left[\int_{0}^{\infty} x f(x) d x-\int_{q}^{\infty} x f(x) d x\right]=\frac{1}{p \mu}\left[\mu-\int_{q}^{\infty} x f(x) d x\right]
$$

and

$$
L(p)=\frac{1}{\mu} \int_{0}^{q} x f(x) d x=\frac{1}{\mu}\left[\int_{0}^{\infty} x f(x) d x-\int_{q}^{\infty} x f(x) d x\right]=\frac{1}{\mu}\left[\mu-\int_{q}^{\infty} x f(x) d x\right]
$$

respectively or equivalently

$$
B(p)=\frac{1}{p \mu} \int_{0}^{p} F^{-1}(x) d x
$$

and

$$
L(p)=\frac{1}{\mu} \int_{0}^{p} F^{-1}(x) d x
$$

respectively, where $\mu=E(x)$ and $q=F^{-1}(p)$.

The Bonferroni and Gini indices are thus defined as

$$
B=1-\int_{0}^{1} B(p) d p
$$

and

$$
B=1-2 \int_{0}^{1} L(p) d p
$$

respectively.

Using pdf of ATPSD (2.1), we get

$$
\int_{q}^{\infty} x f_{6}(x ; \theta, \alpha) d x=\frac{\left\{\theta^{3}\left(\alpha q^{3}+\alpha q^{2}+q\right)+\theta^{2}\left(3 \alpha q^{2}+2 \alpha q+1\right)+2 \alpha \theta(3 q+1)+6 \alpha\right\} e^{-\theta q}}{\theta\left(\theta^{2}+\alpha \theta+2 \alpha\right)}
$$

Now using equation (5.9), (5.5) and (5.6), we get

$$
B(p)=\frac{1}{p}\left[1-\frac{\left\{\theta^{3}\left(\alpha q^{3}+\alpha q^{2}+q\right)+\theta^{2}\left(3 \alpha q^{2}+2 \alpha q+1\right)+2 \alpha \theta(3 q+1)+6 \alpha\right\} e^{-\theta q}}{\theta^{2}+2 \alpha \theta+6 \alpha}\right]
$$


and

$$
L(p)=1-\frac{\left\{\theta^{3}\left(\alpha q^{3}+\alpha q^{2}+q\right)+\theta^{2}\left(3 \alpha q^{2}+2 \alpha q+1\right)+2 \alpha \theta(3 q+1)+6 \alpha\right\} e^{-\theta q}}{\theta^{2}+2 \alpha \theta+6 \alpha}
$$

Now using the equations (5.10) and (5.11) in (5.7) and (5.8), the Bonferroni and Gini indices of ATPSD (2.1) are obtained as

$$
\begin{aligned}
& B=1-\frac{\left\{\theta^{3}\left(\alpha q^{3}+\alpha q^{2}+q\right)+\theta^{2}\left(3 \alpha q^{2}+2 \alpha q+1\right)+2 \alpha \theta(3 q+1)+6 \alpha\right\} e^{-\theta q}}{\theta^{2}+2 \alpha \theta+6 \alpha} \\
& G=\frac{2\left\{\theta^{3}\left(\alpha q^{3}+\alpha q^{2}+q\right)+\theta^{2}\left(3 \alpha q^{2}+2 q+1\right)+2 \alpha \theta(3 q+1)+6 \alpha\right\} e^{-\theta q}}{\theta^{2}+2 \alpha \theta+6 \alpha}-1
\end{aligned}
$$

\section{Estimation of parameters}

In this section, the estimation of parameters of ATPSD using method of moments and method of maximum likelihood have been discussed.

\subsection{Method of Moment Estimates (MOME)}

Since ATPSD (2.1) has two parameters to be estimated, the first two moments about the origin are required to estimate its parameters using method of moments. Equating the population mean to the sample mean, we have

$$
\begin{gathered}
\bar{x}=\frac{\theta^{2}+2 \alpha \theta+6 \alpha}{\theta\left(\theta^{2}+\alpha \theta+2 \alpha\right)}=\frac{\theta^{2}+\alpha \theta+2 \alpha}{\theta\left(\theta^{2}+\alpha \theta+2 \alpha\right)}+\frac{\alpha(\theta+4)}{\theta\left(\theta^{2}+\alpha \theta+2 \alpha\right)} \\
\bar{x}=\frac{1}{\theta}+\frac{\alpha(\theta+4)}{\theta\left(\theta^{2}+\alpha \theta+2 \alpha\right)} \\
\left(\theta^{2}+\alpha \theta+2 \alpha\right)=\frac{\alpha(\theta+4)}{\theta \bar{x}-1}
\end{gathered}
$$

Again equating the second population moment with the corresponding sample moment, we have

$$
\begin{gathered}
m_{2}^{\prime}=\frac{2\left(\theta^{2}+3 \alpha \theta+12 \alpha\right)}{\theta^{2}\left(\theta^{2}+\alpha \theta+2 \alpha\right)}=\frac{2\left(\theta^{2}+\alpha \theta+2 \alpha\right)}{\theta^{2}\left(\theta^{2}+\alpha \theta+2 \alpha\right)}+\frac{4 \alpha(\theta+5)}{\theta^{2}\left(\theta^{2}+\alpha \theta+2 \alpha\right)} \\
m_{2}^{\prime}=\frac{2}{\theta^{2}}+\frac{4 \alpha(\theta+5)}{\theta^{2}\left(\theta^{2}+\alpha \theta+2 \alpha\right)} \\
\theta^{2}+\alpha \theta+2 \alpha=\frac{4 \alpha(\theta+5)}{m_{2}^{\prime} \theta^{2}-2}
\end{gathered}
$$

Equations (6.1) and (6.2) give the following cubic equation in $\theta$

$$
\alpha m_{2}^{\prime} \theta^{3}+4\left(m_{2}^{\prime}-\bar{x}\right) \theta^{2}-2 \alpha(10 \bar{x}-1) \theta+12 \alpha=0 .
$$

Solving equation (6.3) using any iterative method such as Newton-Raphson method, Regula-Falsi method or Bisection method, method of moment estimation(MOME) $\tilde{\theta}$ of $\theta$ can be obtained and substituting the value of $\tilde{\theta}$ in equation (6.1), MOME $\tilde{\alpha}$ of $\alpha$ can be obtained as

$$
\tilde{\alpha}=\frac{(1-\tilde{\theta} \bar{x})(\tilde{\theta})^{2}}{(\tilde{\theta})^{2} \bar{x}-\tilde{\theta}+2(\bar{x}-1)(\tilde{\theta})-6}
$$




\subsection{Maximum likelihood estimates(MLE)}

Let $\left(x_{1}, x_{2}, \cdots, x_{n}\right)$ be random sample from ATPSD (2.1). The likelihood function $L$ is given by

$$
L=\left(\frac{\theta^{3}}{\theta^{2}+\alpha \theta+2 \alpha}\right) \prod_{i=1}^{n}\left(1+\alpha x_{i}+\alpha x_{i}^{2}\right) e^{-n \theta \bar{x}} .
$$

The natural log likelihood function is thus obtained as

$$
\ln L=n\left[3 \ln \theta-\ln \left(\theta^{2}+\alpha \theta+2 \alpha\right)\right]+\sum_{i=1}^{n} \ln \left(1+\alpha x_{i}+\alpha x_{i}^{2}\right)--n \theta \bar{x}
$$

where $\bar{x}$ is the sample mean.

The maximum likelihood estimate(MLE's) $(\hat{\theta}, \hat{\alpha})$ of $(\theta, \alpha)$ are then the solutions of the following non-linear equations

$$
\begin{gathered}
\frac{\partial \ln L}{\partial \theta}=\frac{3 n}{\theta}-\frac{n(2 \theta+\alpha)}{\theta^{2}+\alpha \theta+2 \alpha}-n \bar{x}=0 \\
\frac{\partial \ln L}{\partial \theta}=-\frac{n(\theta+2)}{\theta^{2}+\alpha \theta+2 \alpha}+\sum_{i=1}^{n} \frac{\left(x_{i}+x_{i}^{2}\right) x_{i}}{1+\alpha x_{i}+\alpha x_{i}^{2}}=0
\end{gathered}
$$

These two natural log likelihood equations do not seem to be solved directly, because they cannot be expressed in closed forms. The (MLE's) $(\hat{\theta}, \hat{\alpha})$ of $(\theta, \alpha)$ can be computed directly by solving the natural log likelihood equation using Newton-Raphson iteration available in R-software till sufficiently close values $\hat{\theta}$ of $\hat{\alpha}$ are obtained. The initial values of parameters $\theta$ and $\alpha$ are the MOME $(\hat{\theta}, \hat{\alpha})$ of the parameters $(\theta, \alpha)$.

\section{Applications}

In this section the goodness of fit of ATPSD using maximum likelihood estimation has been discussed with the following two real lifetime datasets.

Data set 1: This data set represents the lifetime's data relating to relief times (in minutes) of 20 patients receiving an analgesic and reported by Gross and Clark [14].

$$
\begin{array}{llllllllllllllllllll}
1.1 & 1.4 & 1.3 & 1.7 & 1.9 & 1.8 & 1.6 & 2.2 & 1.7 & 2.7 & 4.1 & 1.8 & 1.5 & 1.2 & 1.4 & 3 & 1.7 & 2.3 & 1.6 & 2
\end{array}
$$

Data Set 2: The following data represents the failure times (in minutes) for a sample of 15 electronic components in an accelerated life test, available in Lawless [15].

$$
\begin{array}{lllllllllllllll}
1.4 & 5.1 & 6.3 & 10.8 & 12.1 & 18.5 & 19.7 & 22.2 & 23.0 & 30.6 & 37.3 & 46.3 & 53.9 & 59.8 & 66.2
\end{array}
$$

In order to compare lifetime distributions, values of $-2 \ln L$, AIC (Akaike Information Criterion) and K-S Statistic ( Kolmogorov-Smirnov Statistic) for the above data sets have been computed. The formulae for computing AIC and K-S Statistic are as follows:

$A I C=-2 \ln L+2 k$ and $K-S=\sup _{x}\left|F_{n}(x)-F_{0}(x)\right|$, where $k$ is the number of parameters, $n$ is the sample size, and the $F_{n}(x)$ is empirical distribution function. The $\operatorname{MLE}(\hat{\theta}, \hat{\alpha})$ along with their standard errors, S.E $(\hat{\theta}, \hat{\alpha}),-2 \ln L+2 k$, AIC and K-S statistics and p-value of the fitted distributions are presented in the Table 5.

The best distribution is the distribution which corresponds to the lower values of $-2 \ln L$, AIC, and K-S statistic. It is obvious from the goodness of fit test that ATPSD gives much closer fit over two parameters QSD, TPSD and NTPSD and one parameter Sujatha, Lindley and exponential distributions.

\section{Concluding remarks}

Another two-parameter Sujatha distribution (ATPSD) has been introduced which includes exponential distribution and Sujatha distribution as particular cases. Moments about origin and moments about mean of ATPSD have been obtained. The behaviors of coefficient of variation, coefficient of skewness, coefficient of kurtosis, Index of dispersion, hazard rate function and mean residual life function of ATPSD have been discussed with varying values of the parameters. The stochastic ordering, mean deviations, Bonferroni and Lorenz curves, and stress-strength reliability of ATPSD have been discussed. Both method of moment and the method of maximum likelihood have been discussed for estimating parameters. Finally, two examples of observed real lifetime datasets have been presented to show the applications and goodness of fit of ATPSD over two parameters QSD, TPSD and NTPSD and one parameter Sujatha, Lindley and exponential distributions and it has been observed that ATPSD gives much closer fit. 
Table 5: MLE's, S.E $(\hat{\theta}, \hat{\alpha}),-2 \ln L$, AIC and K-S statistics and p-value of the fitted distributions of the given datasets

\begin{tabular}{|l|l|l|l|l|l|l|l|}
\hline Dataset & Distrubition & MLE's & S.E & $-2 \log L$ & AIC & K-S & P-value \\
\hline 1 & ATPSD & $\hat{\theta}=1.35, \hat{\alpha}=25.79$ & $0.18 ; 37.26$ & 50.22 & 54.22 & 0.300 & 0.0551 \\
\hline 1 & NTPSD & $\hat{\theta}=1.06, \hat{\alpha}=42.47$ & $0.165 ; 69.76$ & 52.74 & 56.74 & 0.324 & 0.0298 \\
\hline 1 & TPSD & $\hat{\theta}=0.59, \hat{\alpha}=103.48$ & $0.14 ; 171.61$ & 66.50 & 70.50 & 0.448 & 0.0007 \\
\hline 1 & QSD & $\hat{\theta}=0.56, \hat{\alpha}=104.85$ & $0.131 ; 170.04$ & 66.21 & 70.21 & 0.442 & 0.0008 \\
\hline 1 & Sujatha & $\hat{\theta}=1.136$ & 0.15 & 57.50 & 59.5 & 0.359 & 0.0116 \\
\hline 1 & Lindley & $\hat{\theta}=0.82$ & 0.14 & 60.50 & 62.50 & 0.391 & 0.0044 \\
\hline 1 & Exponential & $\hat{\theta}=0.53$ & 0.12 & 65.67 & 67.67 & 0.440 & 0.0009 \\
\hline \hline 2 & ATPSD & $\hat{\theta}=0.084, \hat{\alpha}=0.007$ & $0 . .19 ; 0.009$ & 128.02 & 132.02 & 0.107 & 0.9890 \\
\hline 2 & NTPSD & $\hat{\theta}=0.088, \hat{\alpha}=30.18$ & $0.018 ; 43.30$ & 129.75 & 133.75 & 0.131 & 0.9300 \\
\hline 2 & TPSD & $\hat{\theta}=0.094, \hat{\alpha}=49.77$ & $0.016 ; 52.97$ & 128.59 & 132.59 & 0.199 & 0.5240 \\
\hline 2 & QSD & $\hat{\theta}=0.084, \hat{\alpha}=12.264$ & $0.02 ; 15.334$ & 128.02 & 132.02 & 0.107 & 0.9890 \\
\hline 2 & Sujatha & $\hat{\theta}=0.107$ & 0.159 & 132.87 & 134.87 & 0.178 & 0.6661 \\
\hline 2 & Lindley & $\hat{\theta}=0.070$ & 0.013 & 128.81 & 130.81 & 0.110 & 0.9836 \\
\hline 2 & Exponential & $\hat{\theta}=0.036$ & 0.009 & 129.48 & 131.48 & 0.156 & 0.8068 \\
\hline
\end{tabular}

\section{Acknowledgements}

Authors are grateful to the editor in chief of the journal and two anonymous reviewers for their constructive comments which improved both the quality and presentation of the paper.

\section{References}

[1] D.V. Lindley, Fiducial distributions and Bayes' theorem, J. R. Stat. Soc. Ser. B Stat. Methodol., 20 (1958), $102-107$.

[2] M.E., Ghitany, B. Atieh, S. Nadarajah, Lindley distribution and its Application, Math. Comput. Simulation, 78 (2008), $493-506$.

[3] R. Shanker, F. Hagos, On modeling of lifetimes data using exponential and Lindley distribution, Biometrics Biostat. Internat. J., 2(4) (2015), 1-5.

[4] R. Shanker, Sujatha distribution and its applications, Statist. Transition New Ser., 17(3) (2016), 1-20.

[5] R. Shanker, The discrete Poisson-Sujatha distribution, Internat. J. Probab. Stat., 5(1) (2016), 1-9.

[6] R. Shanker, F. Hagos, Zero-Truncated Poisson-Sujatha distribution with Applications, J. Ethiopian Statist. Assoc., 24 (2015), $55-63$.

[7] R. Shanker, F. Hagos, Size-biased Poisson-Sujatha distribution with Applications, Amer. J. Math. Statist., 6(4) $2016,145-154$.

[8] R. Shanker, A quasi Sujatha distribution, Internat. J. Probab. Stat., 5(4) (2016), 89-100.

[9] T. Mussie, R. Shanker, A two-Parameter Sujatha distribution, Biometrics Biostat. Internat. J., 7(3) (2018), $188-197$.

[10] T. Mussie, R. Shanker, A New two-Parameter Sujatha distribution with properties and applications, Türkiye Klinikleri J. Biostat., 10(2) (2018), 96-113.

[11] R. Shanker, Akash distribution and its Applications, Internat. J. Probab. Stat., 4(3) (2015), 65-75.

[12] M. Shaked, J.G. Shanthikumar, Stochastic Orders and Their Applications, Academic Press, New York, 1994.

[13] C.E. Bonferroni, Elementi di Statistca generale, Seeber, Firenze, 1930.

[14] A.J. Gross, V.A Clark, Survival Distributions: Reliability Applications in the Biometrical Sciences, John Wiley, New York, 1975.

[15] J.F., Lawless, Statistical Models and Methods for Lifetime data, John Wiley and Sons, New York, 2003. 